\title{
1dentificação da melhor escolha de funcionário para realização de inspeção em estatais do setor elétrico
}

\author{
Roberto Paulo da Silva Pinto Juniora, João Carlos Correia Baptista Soares de Mello ${ }^{\mathrm{b} *}$ \\ arobertjr@furnas.com.br, UFF, Brasil \\ b*jcsmello@pq.cnpq.br, UFF, Brasil
}

\section{Resumo}

Este artigo trata de um mecanismo de escolha de envio de funcionário para a realização de serviços de inspeção de equipamentos adquiridos com base no método multicritério Electre 11. Para tal, é utilizado um exemplo prático em uma das maiores empresas do setor elétrico brasileiro e uma das maiores empresas estatais do Brasil.

Palavras-chave

Electre 11. Inspeção. Viagem. Setor elétrico.

\section{Introdução}

0 setor elétrico brasileiro possui empresas estatais e privadas concorrendo entre si. A eficiência das estatais é cobrada não só pelo governo, mas também pelo próprio mercado, pois a estatal precisa ser suficientemente eficiente para se manter no mercado, minimizando seus custos.

Um fato comum nas empresas estatais no Brasil é a obrigatoriedade da realização de pregão para a aquisição de equipamentos. Tal método de compra propicia inúmeras vantagens, como a equidade de condições de competição entre todos os concorrentes e a fácil entrada no mercado de novos fornecedores, porém também possui desvantagens, como a de permitir que fornecedores inidôneos ou sem condições técnicas participem do processo licitatório.

0 processo licitatório funciona da seguinte forma: a empresa estatal sinaliza para a sociedade que em uma data futura estipulada irá realizar pregão para a aquisição de um determinado equipamento, as empresas interessadas em fornecer o equipamento em questão se inscrevem para participar do pregão, na data e hora estipuladas há o início do pregão, que é vencido pela empresa que cobrar o menor valor pelo equipamento.

Participam do processo licitatório empresas situadas em inúmeras cidades do território nacional e até empresas situadas no exterior através de representantes no Brasil.
Como a empresa estatal não pode escolher a empresa fornecedora de equipamentos, é feita uma série de exigências técnicas e de qualidade do material, aos quais os fornecedores tomam conhecimento antes do processo licitatório. Tais exigências serão verificadas antes do fornecimento e após se saber qual fornecedor é o vencedor do pregão.

Por este motivo, as empresas estatais possuem geralmente departamento de inspeção, responsável por avaliar se o material que será fornecido atende aos pré-requisitos técnicos estabelecidos no processo licitatório; essa análise é realizada nas instalações da empresa vencedora do leilão ou em local escolhido por ela.

Grandes empresas estatais possuem núcleos de inspeção em diferentes localidades. Como exemplo, pode-se citar a maior estatal do setor elétrico brasileiro, cujo departamento de inspeção tem núcleos localizados no Rio de Janeiro, em São Paulo, Campinas e Belo Horizonte. Onde podemos concluir que os custos de viagem e estadia serão diferentes em função da localização do fornecedor vencedor do pregão.

A escolha do inspetor a ser enviado às instalações do fornecedor para verificar se o material atende aos requisitos exigidos leva em consideração fatores como: o conhecimento do material analisado, a experiência do inspetor e principalmente os custos de viagem e estadia. 
Será apresentado neste artigo um modelo baseado no método multicritério Electre 11 para escolher o funcionário mais indicado para a realização da inspeção.

\section{Apoio multicritério a decisão (AMD)}

Os métodos do apoio multicritério à decisão tem um caráter científico e, ao mesmo tempo, subjetivo, trazendo consigo a capacidade de agregar, de maneira ampla, todas as características consideradas importantes inclusive as não quantitativas, com a finalidade de possibilitar a transparência e a sistematização do processo referente aos problemas de tomada de decisão.

0 apoio multicritério à decisão pode ser definido como a atividade do analista de decisões que, baseado em modelos claramente apresentados, ajuda na obtenção de elementos de resposta às questões de um agente de decisão no decorrer de um processo.

Segundo Gomes, Gomes e Almeida (2002) e Gomes, Gonzalez Araya e Carignano (2004), o apoio multicritério à decisão (AMD) pode ser definido como o conjunto de técnicas que têm a finalidade de investigar um número de alternativas, sob múltiplos critérios e objetivos em conflito. É possível gerar soluções, compromisso e uma hierarquização das alternativas, de acordo com o grau de atração destas para o tomador de decisão.

Segundo Bouyssou (1990), uma abordagem multicritério apresenta as seguintes vantagens:

- Torna viável a construção de uma base para o diálogo entre analistas e decisores, que fazem uso de diversos pontos de vista comuns;

- Provê facilidade em incorporar incertezas aos dados sobre cada ponto de vista;

- Permite encarar cada alternativa como um compromisso entre objetivos em conflito.

- Decisor é o indivíduo que proporciona o juízo de valor final que poderá ser usado no momento de avaliar as alternativas disponíveis, com o objetivo de identificar a melhor escolha. Analista é a pessoa encarregada de modelar o problema e, eventualmente, fazer as recomendações relativas à seleção final (SOARES DE MELLO et al., 2003).

A exaustividade das alternativas supõe que, se o decisor introduz uma nova alternativa ao conjunto de escolhas, deverá reformular o modelo com o novo conjunto.

Um critério é uma função que reflete as preferências do decisor quanto a um atributo. Essa função deve ser tal que permita descrever o resultado da comparação entre duas alternativas a e b, segundo um atributo j, a partir da comparação de dois números reais uj(a) e uj(b) (BARBA-ROMERO; POMEROL, 1997; DIAS, 2002).
Portanto, um critério pode ser visto como um modelo, segundo o qual é possível fundamentar uma proposição do tipo:

$\mathrm{uj}(\mathrm{a})>\mathrm{uj}(\mathrm{b}) \Leftrightarrow \mathrm{aPjb}$

$\mathrm{Na}$ qual $\mathrm{Pj}$ representa uma relação binária que expressa que "a é preferivel a b em relação ao atributo j".

Pesos: para o decisor alguns atributos terão maior importância que outros. A medida da importância relativa dos atributos terá maior importância que outros. A medida da importância relativa dos atributos para o decisor denomina-se peso ou ponderação.

Segundo Gomes, Gonzalez Araya e Carignano (2004), uma das seguintes problemáticas é abordada pela decisão multicritério discreta (DMD):

- Problema tipo $\alpha(\mathrm{P} \alpha)$ : seleciona a melhor alternativa ou as melhores alternativas;

- Problema tipo $\beta$ (P $\beta$ ): aceita alternativas que parecem boas e descarta as que parecem ruins, ou seja, realiza uma classificação das alternativas;

- Problema tipo $\gamma(\mathrm{P} \gamma)$ : gera uma ordenação das alternativas;

- Problema tipo $\delta$ (P $\delta$ ): realiza uma descrição das alternativas.

Neste artigo será realizada a análise do problema tipo $\gamma$.

Os primeiros métodos da Escola Francesa de Apoio Multicritério à Decisão foram os da família Electre (Elimination Et Choix Traduisant la Réalité), o Electre 1, proposto por Roy (1968) e, em seguida, o Electre 11, proposto por Roy e Bertier (1973). Tais métodos definem uma série de processos sobre as alternativas consideradas, as quais pertencem ao conjunto de possíveis soluções do problema de decisão analisado.

Uma das principais características introduzidas pelos métodos da família Electre corresponde a um novo conceito do modelo de preferências, que pretende ser uma representação mais realista que o utilizado na teoria de decisão. Esse sistema é apresentado na Tabela 1, retirada de Gomes, Gonzalez Araya e Carignano (2004).

A ideia de incomparabilidade não significa que o agente de decisão esteja excluído do processo de comparação entre duas alternativas que podem ser parte do seu conjunto solução. A relação $\mathrm{aRb}$, ou incomparabilidade entre a e b, significa que o agente de decisão não obteve informações suficientes para definir os valores das alternativas a e $b$, fato este que não pode ser interpretado como indiferença (alb). Por outro lado, a noção de preferência fraca $(\mathrm{aQb})$ significa que o agente de decisão está convicto de que a alternativa b não é preferível à alternativa a, ou seja, não $\mathrm{bPa}$, mas o agente reluta entre $\mathrm{aPb}$ e alb. 
Tabela 1. Situações fundamentais quando se comparam duas alternativas.

\begin{tabular}{clcc}
\hline \multicolumn{1}{c}{ Situações fundamentais quando se comparam duas alternativas } & \multicolumn{1}{c}{ Definição } & \multicolumn{1}{c}{ Relação binária } \\
\hline Situação & \multicolumn{1}{c}{$\begin{array}{c}\text { Existem razões claras e positivas que justificam a } \\
\text { equivalência entre duas alternativas. }\end{array}$} & 1: Simétrica (reflexiva) \\
\hline Preferência estrita & $\begin{array}{l}\text { Existem razões claras e positivas que justificam uma } \\
\text { preferência significativa a favor de uma (bem definida) das } \\
\text { duas alternativas. }\end{array}$ & $\begin{array}{l}\text { Existem razões claras e positivas que não implicam uma } \\
\text { preferência estrita a favor de uma (bem definida) das duas } \\
\text { alternativas. No entanto, essas razões são insuficientes para } \\
\text { que seja assumida uma preferência estrita a favor da outra } \\
\text { ou uma indiferença entre as alternativas. }\end{array}$ & Q: Assimétrica (irreflexiva) \\
\hline Preferência fraca & $\begin{array}{l}\text { Não existem razões claras e positivas que justifiquem uma } \\
\text { das três situações acima. }\end{array}$ & R: Simétrica (irreflexiva) \\
\hline Incomparabilidade & &
\end{tabular}

Outro conceito importante refere-se à transitividade nas relações de preferência. Na teoria da decisão clássica, é comum considerar transitivas as relações P e l, isto é, alb e blc.

Uma forma de delimitar com maior precisão as situações de preferência consiste em estabelecer alguns parâmetros que funcionam como limites de tolerância para a transição de uma situação de preferência a outra, quando duas alternativas são comparáveis. 0 axioma clássico da comparabilidade completa e transitividade cede lugar ao da comparabilidade parcial, que define as relações entre duas alternativas como uma das quatro relações descritas no sistema fundamental de relações de preferência.

Os métodos Electre utilizam a combinação de superação. 0 uso do conceito de superação nos métodos mencionados tenta capturar aquelas relações de preferência que estão bem definidas nas relações apresentadas pelo agente de decisão.

\subsection{Método Electre 1}

Os métodos Electre fazem parte dos denominados métodos de superação, pois eles têm como conceito teórico central as relações de superação. Esses métodos diferenciam-se entre si pela problemática que tentam resolver, pelas informações inter e intracritérios utilizadas e pela quantidade de relações de superação construídas e pesquisadas.

Os métodos Electre consideram os pesos como uma medida da importância que cada critério tem para o decisor, e não como uma taxa marginal de substituição, visto que as avaliações de cada alternativa nos diferentes critérios não se reúnem em uma avaliação global. Esses métodos empregam a informação dos pesos com a finalidade de construir coeficientes de concordância e de discordância.

0 que se procura identificar, no contexto de um problema de decisão, é se existe ou não uma relação de dominância entre duas alternativas, ou seja, se o risco de considerar verdadeira a afirmação "a alternativa a é pelo menos tão boa quanto a alternativa b" é aceitável. As considerações que conduzem à aceitação da relação aSb (a alternativa a supera a alternativa b) podem ser expressas por dois conceitos:

- Concordância - ocorre quando um subconjunto significativo dos critérios considera a alternativa a (fracamente) preferível à b;

- Discordância - ocorre quando não há critérios em que a intensidade da preferência da alternativa $b$ em relação a a ultrapasse um limite inaceitável.

Deve-se definir um limite de concordância c (relativamente grande) e um limite de discordância d (relativamente pequeno) a fim de estabelecer a relação de superação da seguinte forma:

aSb se, e somente se, $\left\{\begin{array}{l}C(a, b) \geq c \\ D(a, b) \leq d\end{array}\right.$

As relações binárias, como as construídas quando se aplicam os métodos Electre, podem ter uma representação equivalente ao serem utilizados grafos orientados (também conhecidos como direcionados). Os grafos orientados são representações gráficas formadas por pontos, denominados vértices, que se relacionam por linhas orientadas nomeadas arcos.

É possível generalizar as etapas dos métodos Electre como:

- A obtenção das avaliações do(s) agente(s) de decisão para as diversas alternativas em relação aos critérios;

- A construção das relações de superação;

- A exploração das relações de superação com o objetivo de selecionar um conjunto de alternativas dominantes ou de ordenar o conjunto de alternativas segundo a sua dominância.

Método Electre 1, o primeiro da família dos métodos de superação, tenta resolver a problemática $\alpha$ (P $\alpha$-esclarecer a decisão por intermédio da escolha de um subconjunto que seja restrito o máximo possível e que contenha as melhores alternativas). Esse método pode ser considerado uma 
generalização do método de Condorcet (SOARES DE MELLO; QUINTELLA; SOARES DE MELLO, 2004; SOARES DE MELLO et al., 2005b; VALLADARES et al., 2008; CAILLAUX et al., 2011).

Existem duas versões do método Electre 1, designadas por Electre lv e Electre 1s. A primeira substitui a exigência de um limiar de discordância por um limiar de veto, considerado por alguns autores (DIAS; ALMEIDA; CLÍMACO, 1996) como mais fácil de apresentar ao decisor. A segunda versão tem a vantagem de trabalhar com pseudocritérios e, portanto, incorporar aos resultados incerteza ou imprecisão associada aos desempenhos.

\subsection{Método Electre 11}

0 método Electre 11 pode ser considerado um aprimoramento do método Electre 1, sendo seu objetivo a busca pela solução da problemática $\gamma$ (P $\gamma$ - esclarecer a decisão por meio de uma ordenação das alternativas).

0 método Electre 11 foi desenvolvido por Roy e Bertier (1973) e pode ser considerado uma extensão do método Electre 1, uma vez que necessita dos dois gráfos produzidos pelo Electre 1 como dados de entrada, representando uma estrutura de preferência forte e outra fraca. 0 Electre 11 resulta num ranking das alternativas não dominadas, sendo indicado para a problemática de ordenação.

A ideia no Electre 11 é escolher aqueles sistemas preferidos pela maioria dos critérios e que não ultrapassem determinado nível de desconforto ou descontentamento aceito pelo decisor para nenhum dos critérios considerados. Existem duas medidas distintas: a de concordância e a de discordância.

0 Electre 11 estabelece uma ordenação completa sobre um conjunto de alternativas inicialmente consideradas que satisfaça:

- 0 teste da concordância, em que a medida da concordância está acima de um nível mínimo de aceitabilidade; e;

- 0 teste da discordância, em que a medida da discordância está abaixo de um nível máximo tolerável de discordância.

As definições de concordância e discordância do Electre 11 diferem daquelas apresentadas para o Electre 1 (GOICOECHEA; HANSEN; DUCKSTEIN, 1982). Para poder analisar as matrizes de concordância e discordância são definidos valores de referência p e $q$, pertencentes ao intervalo de variação entre 0 e 1 . Esses valores indicam que a concordância desejada deverá ser superior ou igual a $p$ e que $q$ definirá a máxima discordância tolerável.

0 procedimento de ordenação do método Electre 11 constitui-se de dois estágios distintos de pré-ordenação. No primeiro estágio define-se a ordenação progressiva e, no segundo, a regressiva. As duas pré-ordenações obtidas, geralmente, são diferentes, porém próximas. Neste caso, o AD pode escolher a média entre elas, caso contrário, deve-se redefinir o problema e reaplicar o método.

Em síntese, o Electre 11 busca ordenar um conjunto de alternativas da melhor para a pior, sendo desenvolvido para resolver problemas de ordenação. São utilizados os conceitos de concordância e discordância para ordenar o conjunto de alternativas. A ordenação é obtida através de duas pré-ordens construídas a partir das relações de sobreclassificação forte e fraca. 0 método Electre 11 aplica-se a problemas que envolvem ordenação de alternativas.

Miranda e Almeida (2004) e Costa, Costa, e Caiado (2006) e Chaves et al. (2010) apresentam estudos envolvendo aplicações do Electre 11. Soares de Mello et al. (2005a) apresentam uma aplicação do Electre 1 na avaliação do tamanho dos aeroportos portugueses. Gomes (1989) realiza um estudo comparativo entre o Electre 11 e o método TODIM aplicado em uma investigação sobre sistemas alternativos de transporte.

\section{Exemplo prático}

Uma empresa estatal do setor elétrico tornou pública uma licitação para a aquisição de 10.000 isoladores; as empresas que concorreram para o fornecimento, sua localização e o valor cobrado são apresentados na Tabela 2.

Tabela 2. Empresas participantes do pregão e seus custos.

\begin{tabular}{ccc}
\hline Empresa & Localização & Preço unitário (R\$) \\
\hline A & São Gonçalo-RJ & 68 \\
B & Pedreira-SP & 65 \\
C & Maringá-PR & 67 \\
D & Manaus-AM & 64 \\
E & Contagem-MG & 69 \\
\hline
\end{tabular}

Tabela 3. Duração de inspeção.

\begin{tabular}{cc}
\hline Equipamento & Duração média de inspeção \\
\hline Barramento & 1 dia por peça \\
Cabo & 1 dia a cada 10.000 metros \\
Capacitor & 1 dia a cada 5 peças \\
Coluna & 5 dias por peça \\
Disjuntor & 1 dia a cada 10.000 peças \\
Estrutura & 1 dia a cada 2.000 peças \\
lsolador & 2 dias por unidade \\
Painel & 1 dia a cada 5 unidades \\
Para-raios & 5 dias por unidade \\
Reator & 1 dia a cada 2 unidades \\
TC & 1 dia a cada 2 unidades \\
TP & 1 dia a cada 2 unidades \\
TPC & 5 dias por unidade \\
\hline Transformador &
\end{tabular}


Tabela 4. Lista completa de inspetores da empresa estatal.

\begin{tabular}{|c|c|c|c|c|c|c|c|c|}
\hline \multirow[b]{2}{*}{ Inspetor } & \multirow[b]{2}{*}{ Localização (cidade) } & \multirow{2}{*}{$\begin{array}{l}\text { Conhecimento de } \\
\text { isoladores }\end{array}$} & \multicolumn{5}{|c|}{ Custo (R\$) } & \multirow[b]{2}{*}{ Experiência } \\
\hline & & & $\begin{array}{c}\text { Passagem } \\
\text { aérea }\end{array}$ & Hotel & Táxi & Diárias & Total & \\
\hline 1 & Rio de Janeiro & 10 & 750 & $4 \times 150$ & $10 \times 20$ & $5 \times 90$ & 2.000 & 4 \\
\hline 2 & Rio de Janeiro & 6 & 750 & $4 \times 150$ & $10 \times 20$ & $5 \times 90$ & 2.000 & 6 \\
\hline 3 & Rio de Janeiro & 5 & 750 & $4 \times 150$ & $10 \times 20$ & $5 \times 90$ & 2.000 & 10 \\
\hline 4 & Rio de Janeiro & 4 & 750 & $4 \times 150$ & $10 \times 20$ & $5 \times 90$ & 2.000 & 2 \\
\hline 5 & Rio de Janeiro & 3 & 750 & $4 \times 150$ & $10 \times 20$ & $5 \times 90$ & 2.000 & 4 \\
\hline 6 & Rio de Janeiro & 4 & 750 & $4 \times 150$ & $10 \times 20$ & $5 \times 90$ & 2.000 & 5 \\
\hline 7 & Rio de Janeiro & 1 & 750 & $4 \times 150$ & $10 \times 20$ & $5 \times 90$ & 2.000 & 1 \\
\hline 8 & Rio de Janeiro & 1 & 750 & $4 \times 150$ & $10 \times 20$ & $5 \times 90$ & 2.000 & 1 \\
\hline 9 & Rio de Janeiro & 1 & 750 & $4 \times 150$ & $10 \times 20$ & $5 \times 90$ & 2.000 & 1 \\
\hline 10 & Rio de Janeiro & 1 & 750 & $4 \times 150$ & $10 \times 20$ & $5 \times 90$ & 2.000 & 1 \\
\hline 11 & Rio de Janeiro & 1 & 750 & $4 \times 150$ & $10 \times 20$ & $5 \times 90$ & 2.000 & 1 \\
\hline 12 & Rio de Janeiro & 1 & 750 & $4 \times 150$ & $10 \times 20$ & $5 \times 90$ & 2.000 & 1 \\
\hline 13 & Rio de Janeiro & 1 & 750 & $4 \times 150$ & $10 \times 20$ & $5 \times 90$ & 2.000 & 1 \\
\hline 14 & Rio de Janeiro & 1 & 750 & $4 \times 150$ & $10 \times 20$ & $5 \times 90$ & 2.000 & 1 \\
\hline 15 & São Paulo & 3 & 800 & $4 \times 150$ & $10 \times 20$ & $5 \times 90$ & 2.050 & 1 \\
\hline 16 & São Paulo & 1 & 800 & $4 \times 150$ & $10 \times 20$ & $5 \times 90$ & 2.050 & 1 \\
\hline 17 & São Paulo & 1 & 800 & $4 \times 150$ & $10 \times 20$ & $5 \times 90$ & 2.050 & 1 \\
\hline 18 & São Paulo & 1 & 800 & $4 \times 150$ & $10 \times 20$ & $5 \times 90$ & 2.050 & 1 \\
\hline 19 & Campinas & 7 & 670 & $4 \times 150$ & $10 \times 20$ & $5 \times 90$ & 1.920 & 6 \\
\hline 20 & Campinas & 3 & 670 & $4 \times 150$ & $10 \times 20$ & $5 \times 90$ & 1.920 & 1 \\
\hline 21 & Belo Horizonte & 3 & 840 & $4 \times 150$ & $10 \times 20$ & $5 \times 90$ & 2.090 & 1 \\
\hline 22 & Belo Horizonte & 1 & 840 & $4 \times 150$ & $10 \times 20$ & $5 \times 90$ & 2.090 & 1 \\
\hline 23 & Belo Horizonte & 1 & 840 & $4 \times 150$ & $10 \times 20$ & $5 \times 90$ & 2.090 & 1 \\
\hline 24 & Belo Horizonte & 1 & 840 & $4 \times 150$ & $10 \times 20$ & $5 \times 90$ & 2.090 & 1 \\
\hline
\end{tabular}

Tabela 5. Tabela resumida de inspetores.

\begin{tabular}{ccccc}
\hline Inspetor & $\begin{array}{c}\text { Localização } \\
\text { (cidade) }\end{array}$ & $\begin{array}{c}\text { Conhecimento } \\
\text { de isoladores }\end{array}$ & $\begin{array}{c}\text { Custos } \\
\text { (R\$) }\end{array}$ & Experiência \\
\hline 1 & Rio de Janeiro & 10 & 2.000 & 4 \\
5 & Rio de Janeiro & 3 & 2.000 & 4 \\
15 & São Paulo & 3 & 2.050 & 1 \\
19 & Campinas & 7 & 1.920 & 6 \\
\hline
\end{tabular}

Tabela 6. Tabela de ponderação de critérios por equipamento.

\begin{tabular}{cccc}
\hline Equipamento & Conhecimento & Custos & Experiência \\
\hline Barramento & 0,2 & 0,7 & 0,1 \\
Cabo & 0,3 & 0,6 & 0,1 \\
Capacitor & 0,4 & 0,4 & 0,2 \\
Coluna & 0,3 & 0,6 & 0,1 \\
Disjuntor & 0,4 & 0,4 & 0,2 \\
Estrutura & 0,3 & 0,3 & 0,4 \\
Isolador & 0,6 & 0,3 & 0,1 \\
Painel & 0,2 & 0,7 & 0,1 \\
Para-raios & 0,5 & 0,4 & 0,1 \\
Reator & 0,4 & 0,3 & 0,3 \\
TC & 0,3 & 0,5 & 0,2 \\
TP & 0,3 & 0,5 & 0,2 \\
TPC & 0,3 & 0,5 & 0,2 \\
Transformador & 0,8 & 0,1 & 0,1 \\
\hline
\end{tabular}

Tabela 7. Tabela de ponderação de critérios para a avaliação de isoladores.

\begin{tabular}{cccc}
\hline Equipamento & Conhecimento & Custos & Experiência \\
\hline lsolador & 0,6 & 0,3 & 0,1 \\
\hline
\end{tabular}

0 vencedor da licitação foi a empresa $\mathrm{D}$, localizada em Manaus-AM, por cobrar o menor valor para fornecer o equipamento.

É calculado o tempo de duração de inspeção com base nas inspeções anteriores, cuja média é apresentada na Tabela 3.

0 resultado para este caso serão 5 dias de inspeção, pois são 10.000 isoladores e é necessário 1 dia a cada 2.000 peças analisadas.

A seguir é iniciado o processo de escolha do inspetor a ser enviado para realizar a inspeção; tal empresa possui a seguinte lista de inspetores.

Na Tabela 4, a coluna localização é o posto onde o funcionário está locado, a coluna conhecimento de isoladores é o conhecimento estipulado do equipamento em questão (isoladores) elaborado pela chefia direta. A coluna custos significa os custos gerados pelo fato do inspetor se deslocar para realizar a inspeção; ainda na coluna custos foi incluída a subcoluna diárias, pois nas empresas estatais se recebem diárias de viagem. Nessa empresa em questão o funcionário receberá a diária de viagem se percorrer distância superior a $60 \mathrm{~km}$ e precisar sair da cidade de locação; caso a empresa fique na mesma cidade do inspetor ou em uma cidade localizada a menos de $60 \mathrm{~km}$ de distância, o funcionário não receberá diária e não terá gastos de passagens aéreas e estadia, tendo somente gastos de deslocamento com táxis. 
Tabela 8. Tabela com os pesos normalizados.

\begin{tabular}{|c|c|c|c|c|c|c|}
\hline \multirow{3}{*}{ Inspetor } & \multicolumn{2}{|c|}{ Conhecimento (Máx) C1 } & \multicolumn{2}{|c|}{ Custos (R\$) (Min) C2 } & \multicolumn{2}{|c|}{ Experiência (Máx) C3 } \\
\hline & $\mathrm{P}=$ & 0,6 & $\mathrm{P}=$ & 0,3 & $\mathrm{P}=$ & 0,1 \\
\hline & Peso original & Peso normalizado & Peso original & Peso normalizado & Peso original & Peso normalizado \\
\hline 1 & 10 & 0,43 & 0,00050 & 0,249 & 4 & 0,267 \\
\hline 5 & 3 & 0,13 & 0,00050 & 0,249 & 4 & 0,267 \\
\hline 15 & 3 & 0,13 & 0,00049 & 0,243 & 1 & 0,067 \\
\hline 19 & 7 & 0,30 & 0,00052 & 0,259 & 6 & 0,400 \\
\hline Soma & 23 & 1 & 0,00201 & 1 & 15 & 1 \\
\hline
\end{tabular}

Tabela 9. Índice de concordância entre as alternativas.

\begin{tabular}{ccccc}
\hline & $\mathrm{C} 1$ & $\mathrm{C} 2$ & $\mathrm{C} 3$ & Somatório \\
\hline $\mathrm{C} 1,5$ & 0,6 & 0,3 & 0,1 & 1,0 \\
$\mathrm{C} 1,15$ & 0,6 & 0,3 & 0,1 & 1,0 \\
$\mathrm{C} 1,19$ & 0,6 & 0,0 & 0,0 & 0,6 \\
$\mathrm{C} 5,1$ & 0,0 & 0,3 & 0,1 & 0,4 \\
$\mathrm{C} 5,15$ & 0,6 & 0,3 & 0,1 & 1,0 \\
$\mathrm{C} 5,19$ & 0,0 & 0,0 & 0,0 & 0,0 \\
$\mathrm{C} 15,1$ & 0,0 & 0,0 & 0,0 & 0,0 \\
$\mathrm{C} 15,5$ & 0,6 & 0,0 & 0,0 & 0,6 \\
$\mathrm{C} 15,19$ & 0,0 & 0,0 & 0,0 & 0,0 \\
$\mathrm{C} 19,1$ & 0,0 & 0,3 & 0,1 & 0,4 \\
$\mathrm{C} 19,5$ & 0,6 & 0,3 & 0,1 & 1,0 \\
$\mathrm{C} 19,15$ & 0,6 & 0,3 & 0,1 & 1,0 \\
\hline
\end{tabular}

Tabela 10. Matriz de concordância.

\begin{tabular}{cc|cccc}
\hline \multicolumn{1}{c}{} & & 1 & 5 & 15 & 19 \\
\hline \multirow{4}{*}{$\mathrm{C}=$} & 1 & & 1,0 & 1,0 & 0,6 \\
& 5 & 0,4 & & 1,0 & 0,0 \\
& 15 & 0,0 & 0,6 & & 0,0 \\
& 19 & 0,4 & 1,0 & 1,0 & \\
\hline
\end{tabular}

Tabela 11. Índice de discordância entre as alternativas.

\begin{tabular}{cccc|c|c}
\hline & C1 & C2 & C3 & $\begin{array}{c}\text { Valor } \\
\text { máximo }\end{array}$ & $\begin{array}{c}\text { Valor } \\
\text { máximo } \\
\text { /D }\end{array}$ \\
\hline Máx & 0,43 & 0,26 & 0,40 & & \\
Min & 0,13 & 0,24 & 0,07 & & \\
\cline { 1 - 2 } Máx & 0,30 & 0,02 & 0,33 & 0,33 & \\
Min(d) & & & & & \\
\cline { 1 - 3 } d1,5 & $-0,30$ & 0,00 & 0,00 & 0,00 & 0,00 \\
d1,15 & $-0,30$ & $-0,01$ & $-0,20$ & $-0,01$ & $-0,02$ \\
d1,19 & $-0,13$ & 0,01 & 0,13 & 0,13 & 0,40 \\
d5,1 & 0,30 & 0,00 & 0,00 & 0,30 & 0,91 \\
d5,15 & 0,00 & $-0,01$ & $-0,20$ & 0,00 & 0,00 \\
d5,19 & 0,17 & 0,01 & 0,13 & 0,17 & 0,52 \\
d15,1 & 0,30 & 0,01 & 0,20 & 0,30 & 0,91 \\
d15,5 & 0,00 & 0,01 & 0,20 & 0,20 & 0,60 \\
d15,19 & 0,17 & 0,02 & 0,33 & 0,33 & 1,00 \\
d19,1 & 0,13 & $-0,01$ & $-0,13$ & 0,13 & 0,39 \\
d19,5 & $-0,17$ & $-0,01$ & $-0,13$ & $-0,01$ & $-0,03$ \\
d19,15 & $-0,17$ & $-0,02$ & $-0,33$ & $-0,02$ & $-0,05$ \\
\hline
\end{tabular}

0 conhecimento do equipamento é estimado pela chefia direta do setor de inspeção, o tempo de experiência é a quantidade em anos de experiência do funcionário em inspecionar o referido equipamento. 0 conhecimento e a experiência variam de equipamento para equipamento.

Como se trata de uma exemplificação da escolha do inspetor, será utilizada uma lista resumida para facilitar a apresentação dos cálculos realizados, como a lista a seguir (Tabela 5).

A ponderação dos critérios é diferente para cada equipamento, pois em alguns equipamentos o conhecimento do equipamento é mais importante que os custos de inspeção e em outros equipamentos a situação se inverte. A Tabela 6 de relação de ponderação de critérios por equipamento é apresentada a seguir.

Da Tabela 7 de relação de ponderação para cada equipamento, são retirados os valores de ponderação para o equipamento em questão (isoladores).

Neste ponto devem ser normalizados os valores de cada critério para cada inspetor. Como objetivamos escolher o de maior valor, normalizaremos os itens pelo critério de maximização. Para os critérios conhecimento e experiência, quanto maior o seu valor, mais atrativo é o item; para o critério custos, quanto maior o valor, menos atrativo é o item, por isso os itens desse critério serão invertidos da forma $y=1 / x$, onde $x$ é o valor inicial do item e y é o valor final. Ficamos então com a Tabela 8 .

A seguir é apresentada uma planilha com a análise de superação das alternativas analisadas duas a duas e somando os valores de pesos dos critérios quando a primeira alternativa supera a segunda (Tabela 9).

Matriz de índice de concordância é apresentada a seguir (Tabela 10).

Os índices de discordância são calculados a partir da fórmula a seguir.

$$
d_{i, k}=\left(\frac{1}{d}\right)_{j \in D\left(x_{j}, x_{k}\right)} \operatorname{máx} \cdot\left(u_{j}\left(x_{i}\right)-u_{j}\left(x_{k}\right)\right)
$$

Onde: $d=$ máx. j (ci,xk) $\in A$ máx $(u j(x k)-u j(x i))$, para $\mathrm{j}=1, \ldots, \mathrm{n}$.

A seguir é apresentada a matriz de discordância D (Tabela 11). 
Tabela 12. Tabela de índices de concordância e discordância.

\begin{tabular}{c|ccc|cc}
\hline \multirow{2}{*}{ Equipamento } & \multicolumn{3}{c}{ indices de concordância } & \multicolumn{2}{c}{ índice de discordância } \\
\hline & C1 & C2 & C3 & D1 & D2 \\
\hline Barramento & 0,9 & 0,7 & 0,4 & 0,89 & 0,45 \\
Cabo & 0,8 & 0,6 & 0,4 & 0,83 & 0,47 \\
Capacitor & 0,8 & 0,7 & 0,5 & 0,89 & 0,44 \\
Coluna & 0,9 & 0,7 & 0,5 & 0,86 & 0,40 \\
Disjuntor & 0,8 & 0,6 & 0,4 & 0,92 & 0,46 \\
Estrutura & 0,8 & 0,6 & 0,4 & 0,90 & 0,45 \\
Isolador & 0,8 & 0,7 & 0,5 & 0,95 & 0,47 \\
Painel & 0,8 & 0,6 & 0,6 & 0,91 & 0,45 \\
Para-raios & 0,8 & 0,6 & 0,4 & 0,87 & 0,47 \\
Reator & 0,8 & 0,6 & 0,5 & 0,81 & 0,50 \\
TC & 0,8 & 0,7 & 0,5 & 0,90 & 0,49 \\
TP & 0,9 & 0,7 & 0,4 & 0,99 & 0,44 \\
TPC & 0,9 & 0,7 & 0,5 & 0,93 & 0,46 \\
\hline
\end{tabular}

Na matriz de superação forte, os índices de concordância par a par devem ser maiores ou iguais ao índice $\mathrm{C} 1$ e os índices de discordância par a par devem ser inferiores ou iguais ao índice D1 ou os índices de concordância par a par devem ser maiores ou iguais ao índice C2 e os índices de discordância par a par inferiores ou iguais ao índice D2.

$\mathrm{Na}$ matriz de superação fraca, os índices de concordância par a par devem ser maiores ou iguais ao índice C3 e os índices de discordância par a par devem ser inferiores ou iguais ao índice D1.

A Tabela 12 dos índices de concordância e discordância é apresentada a seguir.

Da Tabela 12 retiramos os índices para o isolador, que são apresentados na Tabela 13.

Ficamos então com as matrizes de superação forte e fraca respectivamente (Tabelas 14 e 15).

Os grafos das matrizes de superação forte e fraca são apresentados nas Figuras 1 e 2.

Superpondo os dois grafos ficamos com o seguinte grafo (Figura 3).

Frente às análises efetuadas, fica-se com o seguinte resultado: o funcionário 1 supera os demais funcionários, sendo que supera fracamente o funcionário 19. Por sua vez o funcionário 19 supera os funcionários 5 e 15 . 0 funcionário 5 supera o funcionário 15 fortemente, e o 15 supera o funcionário 5 fracamente, o que pode ser encarado como uma intransitividade, porém, a relação de superação forte prevalece sobre a fraca. Assim, o funcionário mais indicado para realizar essa inspeção seria o funcionário 1; caso esteja indisponível ou já esteja realizando inspeção de outro equipamento, o inspetor seguinte seria enviado, no caso o 19, e assim por diante. A Tabela 16 de colocação final é apresentada a seguir.
Tabela 13. Tabela de índices de concordância e discordância para os isoladores.

\begin{tabular}{cccc|cc}
\hline \multirow{2}{*}{ Equipamento } & \multicolumn{3}{c}{$\begin{array}{c}\text { Índices de } \\
\text { concordância }\end{array}$} & \multicolumn{3}{c}{$\begin{array}{c}\text { 1́ndice de } \\
\text { discordância }\end{array}$} \\
\cline { 2 - 7 } & $\mathrm{C} 1$ & $\mathrm{C} 2$ & $\mathrm{C} 3$ & D1 & D2 \\
\hline 1solador & 0,8 & 0,7 & 0,5 & 0,95 & 0,45 \\
\hline
\end{tabular}

Tabela 14. Matriz de superação forte.

$$
\text { Sforte }=\left|\begin{array}{cccc} 
& 1 & 1 & 0 \\
0 & & 1 & 0 \\
0 & 0 & & 0 \\
0 & 1 & 1 &
\end{array}\right|
$$

Tabela 15. Matriz de superação fraca.

$$
\text { Sfraca }=\left|\begin{array}{cccc} 
& 1 & 1 & 1 \\
0 & & 1 & 0 \\
0 & 1 & & 0 \\
0 & 1 & 1 &
\end{array}\right|
$$

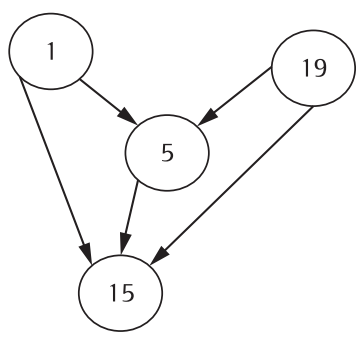

Figura 1. Grafo da matriz de superação forte.

Tabela 16. Colocação final.

\begin{tabular}{cc}
\hline Colocação & Funcionário \\
\hline 1 & 1 \\
2 & 19 \\
3 & 5 \\
4 & 15 \\
\hline
\end{tabular}


Tabela 17. Novo valor de custos.

\begin{tabular}{|c|c|c|c|c|c|c|c|c|}
\hline \multirow{2}{*}{ Inspetor } & \multirow{2}{*}{$\begin{array}{l}\text { Localização } \\
\text { (cidades) }\end{array}$} & \multirow{2}{*}{$\begin{array}{l}\text { Conhecimento de } \\
\text { isoladores }\end{array}$} & \multicolumn{5}{|c|}{ Custos (R\$) } & \multirow{2}{*}{ Experiência } \\
\hline & & & Passagem aérea & Hotel & Táxi & Diárias & Total & \\
\hline 1 & Rio de Janeiro & 10 & 500 & 800 & 200 & 450 & 1950 & 4 \\
\hline 5 & Rio de Janeiro & 3 & 500 & 800 & 200 & 450 & 1950 & 4 \\
\hline 15 & São Paulo & 3 & 0 & 800 & 400 & 450 & 1650 & 1 \\
\hline 19 & Campinas & 7 & 0 & 0 & 200 & & 200 & 6 \\
\hline
\end{tabular}

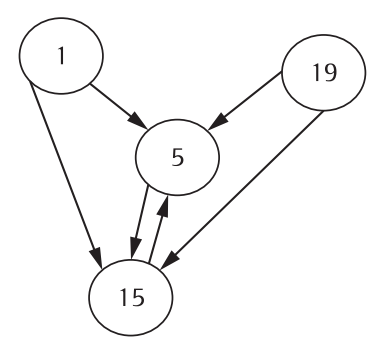

Figura 2. Grafo da matriz de superação fraca.

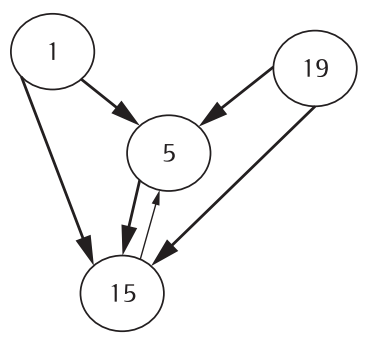

Superação forte $\longrightarrow$ Superação fraca

Figura 3. Grafo das matrizes de superação forte e fraca simultaneamente.

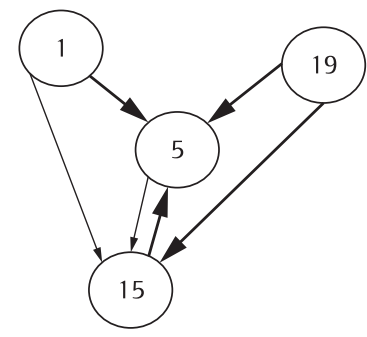

Superação forte $\longrightarrow$ Superação fraca

Figura 4. Grafo final.

Tabela 18. Colocação final.

\begin{tabular}{cc}
\hline Colocação & Funcionário \\
\hline 1 & 19 \\
2 & 1 \\
3 & 15 \\
4 & 5 \\
\hline
\end{tabular}

\section{Segundo exemplo}

Caso a empresa D (localizada em Manaus-AM), vencedora do pregão no exemplo anterior, não tivesse participado do processo ou fosse desclassificada por questões administrativas, o fornecedor que ficou em segundo colocado (segundo menor preço) forneceria 0 equipamento (empresa B localizada em Pedreira-SP).

Os custos seriam o único item a ser alterado em relação ao exemplo anterior, pois foi alterada a cidade de realização da inspeção. A tabela com os novos custos serão apresentados na Tabela 17, para os mesmos funcionários do exemplo anterior.

0 grafo resultante dessa análise é apresentado na Figura 4.

Do grafo anterior pode-se observar que há intransitividade entre os funcionários 1 e 19, pois não há relações de superação entre eles, porém o funcionário 19 supera fortemente todos os outros funcionários enquanto o funcionário 1 supera o funcionário 5 fortemente e o funcionário 15 fracamente. Há outra intransitividade entre os funcionários 5 e 15, porém, pode-se afirmar que o funcionário 15 supera o funcionário 5 porque 0 supera fortemente, enquanto o funcionário 5 supera o funcionário 15 fracamente. A colocação final dos funcionários neste exemplo é a seguinte (Tabela 18):

Em relação ao exemplo anterior pode-se observar alteração entre os dois primeiros e entre os dois últimos colocados.

\section{Conclusões}

Este artigo demonstrou que o método multicritério Electre 11 pode ser uma excelente ferramenta na escolha do funcionário a ser enviado para realizar serviços de inspeção em uma empresa estatal.

Foi apresentada a influência dos custos na escolha de um inspetor de equipamentos, que pode superar fatores como conhecimento e experiência.

No primeiro exemplo, o fornecedor ficou localizado em um lugar neutro, considerado longe para todos os inspetores em questão. No segundo exemplo os custos foram reduzidos para o inspetor locado em Campinas, pois as instalações do fornecedor estão situadas em uma cidade vizinha a Campinas - isto foi um fator determinante para sua escolha.

Nos casos onde ocorreram intransitividade é considerada a relação de superação forte superior a 
relação de superação fraca. Pode haver casos onde a intransitividade possua a mesma relação forte ou fraca, ou ainda não haja relação entre duas opções; nesses casos, as opções ficam em total condição de empate (exceto quando notoriamente uma opção supera com mais intensidade ou em maior número as demais opções), podendo o decisor escolher qualquer uma das opções ou ainda dividir as opções em classes.

Verificou-se que os métodos da família Electre são ideais para este tipo de análise, pois mantém a sequência das alternativas caso um agente seja retirado, o que não é observado em outros métodos multicritério, como no método americano AHP. Nesta análise é primordial que a sequência das alternativas seja constante apesar da saída de alguns agentes, pois o envio de inspetores é uma tarefa corriqueira e muitas vezes os inspetores não estarão disponíveis, pois já estarão realizando outras inspeções, então será efetuado o envio do inspetor subsequente.

Este artigo tratou de uma situação de inspeção no setor elétrico brasileiro, mas tal análise pode ser feita para outros setores, inclusive privados, e outros segmentos de mercado ou em outros países.

\section{Referências}

BARBA-ROMERO, S.; POMEROL, J. C. Decisiones Multicriterio: Fundamentos Teóricos y Utilización Práctica. Madrid: Universidad de Alcalá, 1997.

BOUYSSOU, B. Building criteria: a prerequisite for MCDA. In: BANA E COSTA; C. A. (Org.). Readings in Multiple Criteria Decision Aid. Berlim: Springer Verlag, 1990, p. 58-80. http://dx.doi.org/10.1007/978-3-642-75935-2_4

CAILLAUX, M. A. et al. Container Logistics in Mercosur: Choice of a Transhipment Port Using Ordinal Copeland Method, Data Envelopment Analysis and Probabilistic Composition. Maritime Economics and Logistics, v. 13, n. 4, 2011. PMCid:2799065. http://dx.doi.org/10.1057/mel.2011.20

CHAVES, M. C. C. et al. Utilização do método ELECTRE 11 para avaliação de pilotos no campeonato de Fórmula 1. Produção. v. 20, n. 10, p. 102-113, 2010. http://dx.doi. org/10.1590/S0103-65132010005000012

COSTA, H. G.; COSTA, J. A. B.; CAIADO, J. R. C. Avaliação de eqüinos "Mangalarga Marchador": uma análise multicritério pelo método ELECTRE 11. Pesquisa e Desenvolvimento em Engenharia de Produção, n. 5, p. 1, 2006.
DIAS, L. C. Apontamentos de análise de decisão: como considerar múltiplos critérios. Coimbra: Faculdade de Economia da Universidade de Coimbra, 2002.

DIAS, L. M. C.; ALMEIDA, L. M. A. T.; ClíMACO, J. C. N. Apoio Multicritério à Decisão. Coimbra: Faculdade de Economia da Universidade de Coimbra, 1996.

GOICOECHEA, A.; HANSEN, D.; DUCKSTEIN, L. Multiobjective decision analysis with engeneering and bussiness applications. London: John Wiley and Sons, 1982.

GOMES, L. F. A. M. Comparing two methods for multicriteria ranking of urban transportation system alternatives. Journal of Advanced Transportation, v. 23, n. 2-3, p. 217-219, 1989. http://dx.doi.org/10.1002/ atr.5670230210

GOMES, L. F. A. M.; GOMES, C. F. S. ALMEIDA, A. T. Tomada de Decisão Gerencial Enfoque Multicritério. São Paulo: Editora Atlas, 2002.

GOMES, L. F. A. M.; GONZALEZ ARAYA, CARIGNANO, C. M. Tomada de Decisões em Cenários Complexos. São Paulo: Editora Thompson, 2004.

MIRANDA, C. M. G.; ALMEIDA, A. T. Visão Multicritério da Avaliação de Programas de Pós-Graduação pela CAPES - O Caso da Área Engenharias 111 Baseado nos Métodos ELECTRE 11 e MAUT. Gestão \&t Produção, v. 11, n. 1, p. 51-64, 2004. http://dx.doi.org/10.1590/ S0104-530X2004000100005

ROY, B.; BERTIER, P. La méthode ELECTRE 11 - Une application au média-planning. In: ROSS, M. (Org.). OR' 72. Amsterdam: North-Holland Publishing Company, 1973. p. 291-302.

ROY, B. Classement et choix en présence de points de vue multiples (la méthode ELECTRE). RIRO, v. 8, p. 57-75, 1968.

SOARES DE MELLO, M. H. C. S.; QUINTELLA, H. L. M. M.; SOARES DE MELLO, J. C. C. B. Avaliação do desempenho de alunos considerando classificações obtidas e opiniões dos docentes. Investigação Operacional, v. 24, n. 2, p. 187-196, 2004.

SOARES DE MELLO, J. C. C. B. S. et al. Conceitos básicos do apoio multicritério à decisão e sua aplicação ao projeto Aerodesign. Engevista, v. 5, n. 8, p. 22-35, 2003.

SOARES DE MELLO, J. C. C. B. et al. Avaliação do tamanho de aeroportos portugueses com relações multicritério de superação. Pesquisa Operacional, v. 25, n. 3, p. 313-330, 2005a.

SOARES DE MELLO, J. C. C. B. S. et al. Use of ordinal multi-criteria methods in the analysis of the Formula 1 world championship. Cadernos EBAPE, v. 3, n. 2, p. 3, 2005b.

VALLADARES, G. S. et al. Análise dos componentes principais e métodos multicritério ordinais no estudo de organossolos e solos afins. Revista Brasileira de Ciência do Solo, v. 31, n. 1, p. 285-296, 2008. http://dx.doi.org/10.1590/S010006832008000100027

\section{ldentification of the best selection of employees for the accomplishment of inspection services in the State electric sector Abstract}

This paper presents a mechanism for selection of employees for the accomplishment of inspection services of acquired equipment through the multi-criteria Electre 11 method. To this end, a practical example was used in one of the biggest Brazilian companies in the electric sector and one of the biggest state-owned companies in Brazil.

Keywords

Electre 11. Inspection. Trip. Electric sector. 